\title{
Central nervous system infections in postnerosurgical patients
}

\author{
HL Albornoz ${ }^{1 *}$, L Ibias ${ }^{1}$, A Gadea ${ }^{1}$, F Porcires ${ }^{1}$, R Brinckhaus ${ }^{1}$, N Ramos ${ }^{2}$, H Bagnulo ${ }^{1}$ \\ From International Conference on Prevention \& Infection Control (ICPIC 2011) \\ Geneva, Switzerland. 29 June - 2 July 2011
}

\section{Introduction / objectives}

Central nervous system infections in neurosurgical patients (pts) are a seriuos complication with high morbidity and mortality. We describe characteristics of patients and episodes, microorganisms and evolution of neurosurgical pts with meningitis $(\mathrm{M})$ or ventriculitis $(\mathrm{V})$ in one ICU in Uruguay.

\section{Methods}

Retrospective analysis of neurosurgical pts with $\mathrm{M}$ or $\mathrm{V}$ in a ten year period (2000-2010). $\mathrm{M}$ and $\mathrm{V}$ was defined based in cerebrospinal fluid findings (glucose $<0.4 \mathrm{~g} / \mathrm{L},<40 \%$ plasmatic glucose, leucocytes $>50 / \mathrm{mL}$ ( $>50 \%$ neutrophils), lactate $>4 \mathrm{mM} / \mathrm{L}$ ) and culture (definitive episodes). $\mathrm{V}$ required intraventricular procedure or device implantation.

\section{Results}

69 pts (47 years, male 69\%, SAPS II 33, mechanical ventilation 92\%) developed 77 episodes (M 44, V 32). Neurosurgical diseases were trauma (39\%), meningeal hemorrhage (20\%), intracerebral hemorrhage (17\%), intracranial tumor (12\%). Cerebrospinal fluid leakage was present in 25\%, ventriculostomy in 35\% (catheter permanence 6.2 days), subdural catheter in $30 \%$ (catheter permanence 4.2 days). Microorganisms were mainly Gram negative bacilli (Acinetobacter sp (20, 26\%), Klebsiella sp (7, 9\%), Ps aeruginosa ( $7,9 \%)$, Proteus sp (3,3.9\%), Enterobacter sp (3, 3.9\%), $\mathrm{S}$ aureus $(8,10.4 \%), \mathrm{S}$ coagulase negative (6, $7.8 \%)$, Enterococcus sp (3, 3.9\%), Candida sp $(5,6.5 \%)$ ). Crude mortality was $29 \%(20 / 69)$.

\section{Conclusion}

In a selected group of seriously ill and high risk neurosurgical patients $\mathrm{M}$ and $\mathrm{V}$ were mainly caused for Gram negative bacilli and had high mortality.

${ }^{1}$ Intensive Care Unit, Hospital Maciel, Montevideo, Uruguay

Full list of author information is available at the end of the article

\section{Disclosure of interest}

None declared.

\section{Author details}

${ }^{1}$ Intensive Care Unit, Hospital Maciel, Montevideo, Uruguay. ${ }^{2}$ Infection Control Commitee, Hospital Maciel, Montevideo, Uruguay.

Published: 29 June 2011

doi:10.1186/1753-6561-5-S6-P191

Cite this article as: Albornoz et al:: Central nervous system infections in postnerosurgical patients. BMC Proceedings 2011 5(Suppl 6):P191.
Submit your next manuscript to BioMed Central and take full advantage of:

- Convenient online submission

- Thorough peer review

- No space constraints or color figure charges

- Immediate publication on acceptance

- Inclusion in PubMed, CAS, Scopus and Google Scholar

- Research which is freely available for redistribution

Submit your manuscript at www.biomedcentral.com/submit
C Biomed Central

\section{() Biomed Central}

